\title{
Pruebas monoaurales de habla de baja redundancia: Evaluación de la separación/cierre monoaural
}

\section{Monaural low-redundancy speech tests: Assessment of monoaural separation/closure}

\author{
Anthony Marcotti F. ${ }^{1,2}$, Javier Galaz M. ${ }^{1}$, Felipe Iturriaga O. ${ }^{1}$, Gonzalo Aguilar S. ${ }^{1}$
}

${ }^{1}$ Carrera de Fonoaudiología, Departamento de Ciencias de la Salud, Facultad de Medicina, Pontificia Universidad Católica de Chile. Santiago, Chile

¿Escuela de Fonoaudiología Facultad de Ciencias de la Salud Universidad de Las Américas. Santiago, Chile.

Los autores declaran no tener conflictos de interés.

Recibido el 4 de febrero de 2020. Aceptado el 7 de octubre de 2020

Correspondencia: Anthony Marcotti F. Departamento de Ciencias de la Salud,

Pontificia Universidad Católica de Chile,

Av. Vicuña Mackenna 4860 Macul.

Santiago, Chile. Email: anthony.marcotti@uc.c

\section{Resumen}

La separación y el cierre monoaural son mecanismos del procesamiento auditivo que permiten mantener interacciones comunicativas significativas en ambientes reales, con condiciones acústicas adversas, ruido competitivo e interlocutores no ideales. Estas habilidades dependen de la redundancia intrínseca, que está determinada por las estructuras y fisiología del sistema nervioso auditivo central, y de la redundancia extrínseca, que está determinada por las pistas acústicas, lingüísticas y contextuales de la señal auditiva. Estos procesos se han evaluado a través de distintas formas de degradación de la redundancia extrínseca, dando origen a tres categorías de prueba: pruebas de habla filtrada de pasa baja, pruebas de habla en ruido y pruebas de habla de tiempo comprimido. Últimamente, se han popularizado las pruebas de habla en ruido, sin embargo, las tres categorías cuentan con documentación que avalan su utilidad, se encuentran disponibles en español y es posible aplicarlas tanto en niños como en adultos. A pesar de que algunas cuentan con valores normativos establecidos, es necesario interpretar los resultados considerando su validez ecológica y algunas variables como escolaridad, nivel socioeconómico y otros determinantes sociales de la salud que podrían influenciar el rendimiento.

Palabras clave: separación/cierre monoaural, pruebas de habla filtrada, pruebas de habla en ruido, pruebas de habla de tiempo comprimido.

\begin{abstract}
Monaural separation and closure are auditory processing mechanisms that allow to maintain significant communicative interactions in real environments, with adverse acoustic conditions, competitive noise and non-ideal speakers. These skills depend on intrinsic redundancy, which is determined by the structures and physiology of the central auditory nervous system, and extrinsic redundancy, which is determined by the acoustic, linguistic and contextual cues of the auditory signal. These processes have been assessed through different forms of degradation of extrinsic redundancy, giving rise to three test categories: low-pass filtered speech tests, speech in noise tests and time-compressed speech tests. Speech in noise tests have become popular in recent years, however, all three categories have documentation to support their usefulness, are available in Spanish and can be applied to both children and adults. Although some have established normative values, it is necessary to interpret the results considering their ecological validity and some variables such as schooling, socioeconomic status and other social determinants of health that could influence performance. Keywords: monaural separation/closure, low-pass filtered speech tests, speech in noise tests, time-compressed speech tests.
\end{abstract}

\section{Introducción}

Los seres humanos tienen la capacidad para comprender mensajes auditivos con información lingüística aun cuando estos hayan sido presentados de manera simultánea con señales auditivas irrelevantes (ruido competitivo) o cuando han sufrido algún tipo de degradación (pérdida de información). Esta capacidad ha sido denominada separación/cierre monoau- 
ral o MSC (monoaural separation/closure) $)^{1,2}$ $y$, a pesar de que en muchas ocasiones no se hace diferencia entre "separación" y "cierre", estos serían procesos sutilmente distintos. El primero se refiere a la capacidad de escuchar un estímulo en presencia de otras señales acústicas, es decir, una situación de ruido competitivo. Un ejemplo clásico del despliegue de este proceso se daría al escuchar con éxito a un interlocutor en un restaurante, con música de fondo y con otras personas conversando alrededor. El segundo hace referencia a la habilidad para utilizar la redundancia a favor de una adecuada comprensión del mensaje cuando este ha perdido alguna característica acústica, es decir, una situación con una señal auditiva degradada. Un ejemplo de este proceso es cuando se comprende un mensaje en su globalidad al hablar por teléfono celular con la señal entrecortada y aunque haya perdido segmentos de información. Tanto la separación como el cierre monoaural actuarían en conjunto en ambientes cotidianos, por ejemplo, al conversar por teléfono celular en la calle, con una señal inestable en presencia de ruido vehicular.

La denominación de MSC surge de una serie de análisis factoriales exploratorios y confirmatorios para una batería de pruebas conductuales de procesamiento auditivo realizado por Schow y cols. ${ }^{2,3}$. Los autores concluyeron que solo existen cuatro procesos auditivos medibles conductualmente: patronaje auditivo/ordenamiento temporal (auditory pattern/temporal ordering), separación binaural (binaural separation), integración binaural (binaural integration) y MSC. Esta conclusión contrasta con los seis procesos descritos por la American Speech-Language-Hearing Association (ASHA) y de la división explícita que esta realiza de los subcomponentes del MSC. Para la $\mathrm{ASHA}^{4}$, estos subcomponentes se denominan: 1) desempeño auditivo con señales acústicas competitivas y 2) desempeño auditivo con señales acústicas degradadas. Además, inicialmente la ASHA realizó una clasificación de las pruebas auditivas conductuales disponibles para la evaluación del procesamiento auditivo, siendo las pruebas monoaurales de habla de baja redundancia las encargadas de evaluar estas dos habilidades ${ }^{4}$.

Estas diferencias de nomenclatura produ- cen algunas confusiones al revisar la literatura debido a que tanto la clasificación de Schow y cols. ${ }^{2,3}$ como la de la ASHA $^{5}$ siguen estando vigentes. En términos generales, el desempeño auditivo con señales competitivas y desempeño auditivo con señales acústicas degradadas descritos por la ASHA serían homologables con la separación y cierre monoaurales, respectivamente. De la misma forma, las pruebas para evaluar estas habilidades, denominadas según la ASHA como pruebas monoaurales de habla de baja redundancia, serían las mismas pruebas que otros autores denominan pruebas de MSC $^{2}$. Es necesario aclarar estos conceptos ya que existen importantes textos de referencia como el de Bellis ${ }^{1}$ o el de Geffner \& Ross-Swain ${ }^{6}$ que utilizan ambas terminologías de manera intercambiable.

El objetivo de la presente revisión fue describir los métodos disponibles para la evaluación clínica del MSC y sus consideraciones. Se realizó una revisión narrativa de la literatura de carácter integrativa. La consulta bibliográfica se realizó en octubre del año 2019. Se realizó una consulta inicial en los principales textos de la literatura gris de procesamiento auditivo de los últimos 10 años. Se extrajeron términos relevantes como sinónimos de MSC, nombres de pruebas clínicas y sus clasificaciones para, posteriormente, consultar las bases de datos PubMed y Scielo. Se revisaron artículos sin límite de antigüedad en idioma inglés, portugués y español. Para profundizar los conceptos atingentes se realizó, además, una revisión ancestral, tanto para la literatura gris como para los artículos.

\section{Redundancia intrínseca y extrínseca}

La redundancia hace referencia a dos tipos de recursos con los que cuenta un oyente para lograr una adecuada comprensión del mensaje auditivo. Por un lado, está la redundancia intrínseca, que está determinada por la estructura y fisiología del sistema nervioso auditivo central (SNAC), en donde existen múltiples vías paralelas de transmisión de información ${ }^{7}$. Por otro lado, existe la redundancia extrínseca, que surge de las múltiples características acústicas de la señal como los aspectos temporales, frecuencia e intensidad, 
pero principalmente de las pistas lingüísticas inherentes al lenguaje ${ }^{1}$. La manipulación de este último tipo de redundancia es un factor para considerar en la selección del material verbal en pruebas logoaudiométricas, debido a que el rendimiento esperado en palabras de corta metría y sin una carga lingüística importante (por ejemplo, monosílabos sin sentido) sería mucho menor al esperado en palabras de mayor metría y con información lingüística (por ejemplo, términos familiares) incluso en sujetos con audición normal. En el primer caso, la ausencia de redundancia extrínseca permitiría centrar la evaluación en aspectos casi exclusivamente auditivos, mientras que aumentar la redundancia extrínseca, permitiría agregar mayor validez ecológica a la evaluación (Figura 1). La validez ecológica se refiere a la necesidad de utilizar mediciones que exploren las relaciones entre el rendimiento del sujeto en una prueba determinada y el rendimiento funcional en el mundo real ${ }^{8}$.

Cuando un oyente con redundancia intrínseca conservada (es decir, sin alteraciones en el SNAC) escucha a un hablante con niveles adecuados de redundancia extrínseca, se espera un rendimiento comunicativo normal. Si al mismo oyente se le presenta un material verbal degradado, es decir, con una reducción de la redundancia extrínseca (filtrando el mensaje, agregando competencia o alterando algún otro aspecto físico de la onda sonora), aun así, podría utilizar la redundancia intrínseca para lograr un rendimiento comunicativo normal. Por el contrario, si un oyente presenta alguna alteración en el SNAC, es decir, una reducción de la redundancia intrínseca, su desempeño comunicativo estaría determinado en mayor medida por la redundancia extrínseca, por lo que, si esta última se encuentra adecuada, se podría esperar un rendimiento más o menos eficiente. Sin embargo, si bajo las mismas circunstancias de alteración del SNAC se presenta una señal verbal degradada, las estrategias disponibles serán insuficientes y, por lo tanto, no se logrará un adecuado desempeño comunicativo.

\section{Pruebas monoaurales de habla de baja redundancia}

Es posible degradar la redundancia extrínseca en al menos tres aspectos ${ }^{7}$ :

a) En sus características frecuenciales o espectrales.

b) Introduciendo algún tipo de señal competitiva como ruido de fondo.

c) Alterando la señal en su dominio temporal al comprimir la señal.

Cada una de estas formas de degradación de la señal ha dado origen a una subcategoría distinta de pruebas: pruebas de habla filtrada, pruebas de habla en ruido y pruebas de habla

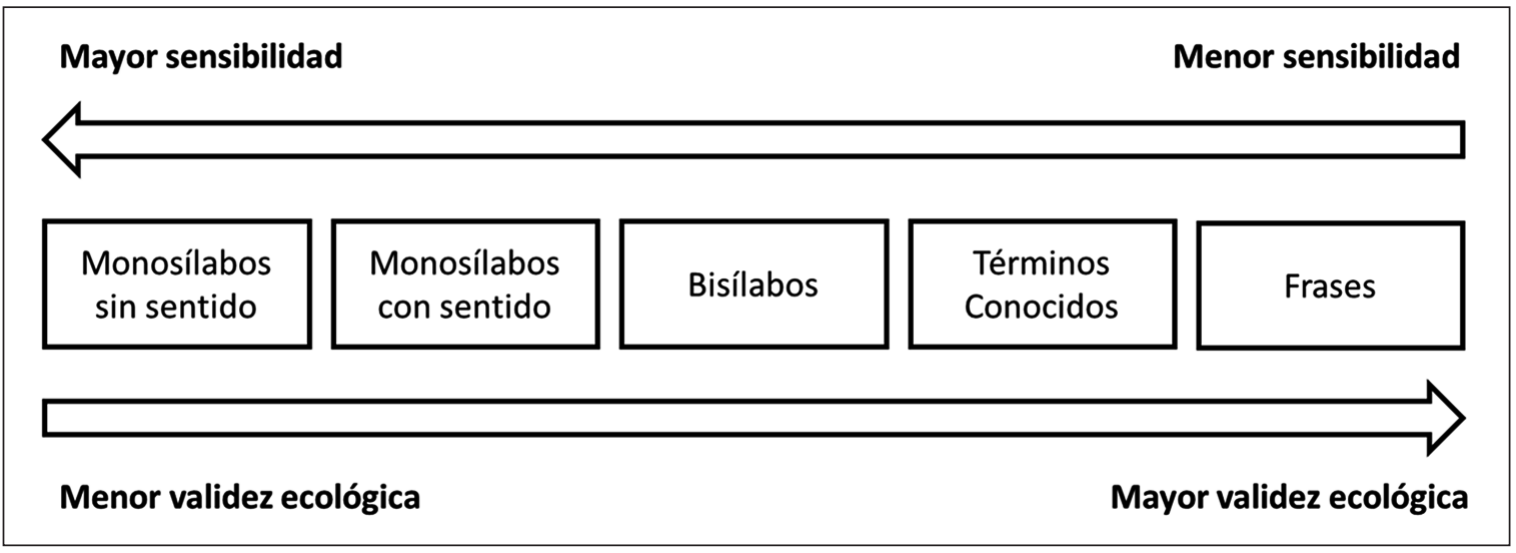

Figura 1. Relación entre sensibilidad y validez ecológica de los materiales verbales más utilizados en las pruebas logoaudiométricas en función de la redundancia extrínseca. Los materiales menos redundantes (monosílabos sin sentido) son más sensibles a lesiones o disfunciones del SNAC, pero tienen menos validez ecológica. Los materiales más redundantes (frases) son menos sensibles, pero proporcionan una mayor validez ecológica. 
de tiempo comprimido, respectivamente. En general, estas pruebas han demostrado ser especialmente útiles para detectar disfunciones a nivel de tronco cerebral y de regiones corticales, y son particularmente sensibles a disfunciones en la corteza auditiva primaria (Tabla 1). Además, existen otras formas de reducir la redundancia extrínseca como la modificación, la intensidad (presentando palabras a baja intensidad), la alteración del dominio temporal en formas diferentes (por ejemplo, agregando silencios en lugares específicos) o agregando reverberancia (manteniendo ciertas características del sonido a pesar de que la fuente lo ha dejado de emitir), sin embargo, estos últimos métodos no son utilizados en pruebas clínicas.

\section{Pruebas de habla filtrada de pasa baja}

Al igual que las pruebas logoaudiométricas convencionales, estas pruebas consisten generalmente en listados de entre 20 a 50 monosílabos por oído que el sujeto evaluado debe repetir. La diferencia con las pruebas clásicas radica en que estos listados se encuentran sometidos a filtros digitales. El filtraje se realiza mediante la eliminación del contenido de alta frecuencia por encima de una frecuencia específica de corte. Dependiendo de la prueba, este filtro de pasa baja puede tener su frecuencia de corte entre 500 y $1000 \mathrm{~Hz}$. Cuanto más baja es la frecuencia de corte, mayor es la dificultad de la tarea. Lo mismo ocurre con la pendiente del filtro, aumentando la dificultad a medida que se vuelve más pronunciada (por ejemplo, un listado de palabras filtradas con una pendiente de $32 \mathrm{~dB} /$ octava sería más complejo que uno de $18 \mathrm{~dB} /$ octava $)^{9}$. Usualmente, estas pruebas son aplicadas entre 40-50 dB SL. La racionalización de estas pruebas es la pérdida de información de tipo espectral principalmente en frecuencias agudas que ocurre, por ejemplo, cuando el interlocutor se tapa la boca con la mano, utiliza mascarilla o, básicamente, cuando existe cualquier tipo de barrera física entre el interlocutor y el oyente.

Históricamente, el primer registro que se tiene de una prueba de habla filtrada de pasa baja (abreviadas generalmente como FS, del inglés filtered speech) y, en general, de alguna prueba monoaural de habla de baja redundancia, data del año 1954. Bocca y cols..$^{10}$ describieron la utilización de una prueba de estas características con un filtro de pasa baja de $800 \mathrm{~Hz}$ capaz de detectar tumores en el lóbulo temporal a través de un rendimiento deficiente en el oído contralateral, aún en presencia de umbrales auditivos normales. Un año más tarde, lograron demostrar que este mismo tipo de pruebas, utilizando un filtro de $1000 \mathrm{~Hz}$, tendría resultados normales en aquellos sujetos con lesiones temporales que no afectaban específicamente las regiones auditivas ${ }^{11}$, por lo que la sensibilidad y especificidad de estas pruebas para este tipo de lesiones sería significativamente mayor a las de una audiometría y una logoaudiometría convencional.

En 1977, se propuso una de las primeras

Tabla 1. Subcategorías de pruebas monoaurales de habla de baja redundancia y sus características

\begin{tabular}{|c|c|c|c|c|}
\hline $\begin{array}{c}\text { Subcategoría de } \\
\text { prueba }\end{array}$ & $\begin{array}{c}\text { Tipo de } \\
\text { degradación }\end{array}$ & $\begin{array}{l}\text { Proceso evaluado } \\
\text { según } \text { ASHA }^{4}\end{array}$ & $\begin{array}{l}\text { Proceso evaluado } \\
\text { según Schow y cols., }\end{array}$ & $\begin{array}{c}\text { Utilidad en } \\
\text { topodiagnóstico }\end{array}$ \\
\hline $\begin{array}{l}\text { Pruebas de habla } \\
\text { filtrada de pasa baja }\end{array}$ & Espectral & $\begin{array}{c}\text { Rendimiento auditivo } \\
\text { con señales acústicas } \\
\text { degradadas }\end{array}$ & Cierre auditivo & $\begin{array}{l}\text { Corteza auditiva } \\
\text { primaria }\end{array}$ \\
\hline $\begin{array}{l}\text { Pruebas de habla en } \\
\text { ruido }\end{array}$ & Espectral & $\begin{array}{c}\text { Rendimiento auditivo } \\
\text { con señales acústicas } \\
\text { competitivas }\end{array}$ & Separación auditiva & $\begin{array}{c}\text { Corteza cerebral } \\
\text { en general y tronco } \\
\text { cerebral bajo }\end{array}$ \\
\hline $\begin{array}{l}\text { Pruebas de habla de } \\
\text { tiempo comprimido }\end{array}$ & Temporal & $\begin{array}{c}\text { Rendimiento auditivo } \\
\text { con señales acústicas } \\
\text { degradadas }\end{array}$ & Cierre auditivo & $\begin{array}{c}\text { Corteza auditiva } \\
\text { primaria, tronco } \\
\text { cerebral }\end{array}$ \\
\hline
\end{tabular}


baterías clínicas para evaluar procesamiento auditivo en niños entre cinco y diez años la cual incluía, entre otras mediciones, una prueba denominada "filtered word" (FW $)^{12}$. Esta prueba consistía en dos listas de 50 palabras de estructura consonante-vocal-consonante. A las palabras se les aplicó un filtro de pasa baja de $500 \mathrm{~Hz}$ con una pendiente de $18 \mathrm{~dB} /$ octava, con una intensidad de presentación de $50 \mathrm{~dB}$ SL. Esta prueba se considera normal cuando el sujeto obtiene un rendimiento superior o igual al $70 \%$ y una asimetría (entre oídos) menor al 10\%. Años más tarde, esta prueba fue modificada para ser incorporada en prácticamente todas las versiones del Screening Test for Auditory Processing (SCAN), batería de pruebas conductuales para realizar tamizaje de procesamiento auditivo en niños y adultos de amplia utilización en Estados Unidos. Dentro de las principales modificaciones, destaca el cambio de filtro a $1000 \mathrm{~Hz}$ y una pendiente de $32 \mathrm{~dB} /$ octava $^{13}$. La misma batería fue adaptada para su utilización en adolescentes y adultos ${ }^{14}$, también incorporando una versión del FW, pero con un filtro en $750 \mathrm{~Hz}$.

A pesar de que la mayoría de las pruebas han sido desarrolladas en inglés, existe una prueba de FS en español perteneciente a la batería de pruebas Santiago APD, comercializada actualmente por Auditec ${ }^{\circledR 15}$. La prueba consiste en dos listados de 25 monosílabos con sentido (las mismas palabras para cada lista, pero en distinto orden) a las cuales se les aplicó un filtro de pasa baja de $500 \mathrm{~Hz}$ con una pendiente de $45 \mathrm{~dB} /$ octava. Estas palabras pertenecen a la lista propuesta por Rosenblüt y De la Cruz ${ }^{16}$. Se recomienda presentar ambos listados a 50 $\mathrm{dB}$ HL sobre el promedio tonal puro o PTA (pure-tone averege) de las frecuencias 500, 1000 y $2000 \mathrm{~Hz}$. La tarea del evaluado es repetir las palabras escuchadas y cada palabra repetida correctamente equivale a un $4 \%$, siendo el total máximo de puntuación un 100\%. En adultos, se espera un rendimiento promedio de un $80 \%$, con una desviación estándar de un $10 \%$, no habiéndose reportado diferencias entre oídos. No existen reportes de la utilización de esta prueba en población pediátrica, sin embargo, las versiones en inglés han sido aplicadas en niños desde los cinco años ${ }^{13}$.

Aunque se han publicado datos limitados de sensibilidad y especificidad para este tipo de pruebas, existe evidencia de que serían más sensibles a lesiones o disfunciones del lóbulo temporal que a lesiones interhemisféricas y del tronco encefálico, presentando un rendimiento deficiente en el oído contralateral a la lesión. Un estudio antiguo documenta una sensibilidad de un $74 \%$ para lesiones de lóbulo temporal, mientras que uno posterior establece una sensibilidad de un 65\% para el mismo tipo de lesiones, $62 \%$ para lesiones de tronco cerebral, $40 \%$ para lesiones vasculares de tronco y de un 56\% para tumores de ángulo pontocerebeloso ${ }^{17}$. En un estudio más reciente, se ha reportado una sensibilidad de un 50\% con una especificidad de un $72 \%$ en pacientes adultos con lesiones corticales ${ }^{18}$. En esta última investigación se documentó que la sensibilidad de este tipo de pruebas aumentaría a un $90 \%$ cuando se utilizan en conjunto con otras pruebas como dígitos dicóticos o patrones de frecuencia, aunque la especificidad disminuiría a un $62 \%$.

\section{Pruebas de habla en ruido}

Este tipo de pruebas consiste en presentar señales verbales a los sujetos evaluados en presencia de distintos tipos de ruido de fondo a distintas relaciones de señal/ruido (SNR). La racionalización subyacente es la mala percepción del habla en ambientes ruidosos, que es uno de los síntomas más comunes del trastorno del procesamiento auditivo ${ }^{19}$. Así, por ejemplo, estas pruebas pretenden reproducir situaciones que enfrenta un menor en la sala de clases cuando pretende prestar atención a la voz del profesor en presencia de ruido de habla de sus compañeros. En numerosos textos de referencia, se destaca la importancia de incluir una prueba de habla en ruido en la batería de evaluación conductual básica de procesamiento auditivo ${ }^{1,5,18}$. A pesar de que los registros de la utilización de ruido en pruebas de reconocimiento verbal datan del año $1947^{20}$, los primeros reportes de su utilidad en sujetos con lesiones del SNAC aparecen en el año $1972^{21}$, donde los investigadores midieron el reconocimiento de estímulos verbales a distintos SNR en pacientes normales, con lesiones de tronco cerebral y con lesiones de lóbulo temporal. El rendimiento de los sujetos con lesiones de tronco fue significativamente 
menor que en los sujetos normales, no asociado a un oído en particular. Sucedió lo mismo en aquellos sujetos con lesiones temporales, sin embargo, en este grupo, el rendimiento inferior se evidenció en el oído contralateral a la lesión. Otros autores, han obtenido resultados similares y han evidenciado deficiencias de habla en ruido en el oído contralateral al hemisferio con lesión cortical ${ }^{22}$.

Dentro de las pruebas más descritas en la literatura, destacan la prueba de palabras en ruido o WIN (words in noise), la prueba de figura auditiva de fondo o AFG (auditory figure ground), la prueba de identificación de oraciones sintéticas con ruido ipsilateral competitivo o SSI-ICM (synthetic sentence identification - ipsilateral competing message) y la prueba de inteligibilidad de habla pediátrica con ruido ipsilateral competitivo PSI-ICM (pediatric speech intelligibility test-ipsilateral competing message). El WIN consiste en un listado de 70 monosílabos, presentados de 7 a 10 grupos con distintos SNR, entre 0 a $24 \mathrm{~dB}$ en intervalos de $4 \mathrm{~dB}^{23}$ utilizando ruido multihablante o babble (ruido conformado por habla de múltiples interlocutores de manera simultánea). El AFG está compuesto por dos listados de 20 monosílabos presentados con ruido babble a un SNR de $8 \mathrm{~dB}$ en la versión pediátrica y a 0 $\mathrm{dB}$ en la versión para adolescentes y adultos ${ }^{14,24}$. El SSI-ICM consiste en una serie de oraciones sin sentido que el paciente debe identificar en un listado escrito de 10 alternativas. Estas se presentan simultáneamente con un ruido de habla continua a un SNR entre 20 a $-20 \mathrm{~dB}^{25,26}$. Por último, el PSI-ICM es la versión pediátrica del SSI-ICM donde, además de frases, se cuenta con monosílabos como estímulos e imágenes para apoyar las respuestas ${ }^{27}$. De estas pruebas, solo el SSI-ICM y el PSI-ICM cuentan con una versión en español ${ }^{28}$, sin embargo, no se ha encontrado literatura que reporte experiencias con estas versiones.

Una de las pruebas disponibles en español, es la prueba de habla en ruido o SIN (speech in noise), también disponible en la batería de Santiago $\mathrm{APD}^{15}$. Utiliza las mismas palabras de Rosenblüt y De la Cruz ${ }^{16}$ que, además, incorpora la prueba FS de la misma batería, pero aleatorizadas en 4 listados distintos de 25 monosílabos (las mismas palabras para cada lista, pero en distinto orden), todos presentados con ruido blanco ipsilateral a distintos SNR. Dos listas se presentan a $10 \mathrm{~dB}$ SNR, una para cada oído. Las otras dos listas constituyen un ítem de mayor dificultad por ser presentadas a $0 \mathrm{~dB}$ SNR y, de la misma forma que las anteriores, corresponde una lista para cada oído. La intensidad de presentación es de $40 \mathrm{~dB}$ HL por sobre el PTA y la tarea del sujeto evaluado es repetir cada una de las palabras escuchadas. Cada palabra repetida correctamente equivale a un $4 \%$, con un desempeño máximo de un $100 \%$. De esta manera, cada oído tendrá dos puntuaciones, una para la condición de $10 \mathrm{~dB}$ SNR y otra para $0 \mathrm{~dB}$ SNR. En adultos, se espera que a $10 \mathrm{~dB}$ SNR el rendimiento promedio sea de $97 \%$ con una desviación estándar de 3\% y a $0 \mathrm{~dB}$ SNR un promedio de $92 \%$ con una desviación estándar de $5 \%{ }^{15}$. No se encontraron experiencias de esta prueba en particular en población pediátrica, sin embargo, algunas pruebas de esta categoría han sido aplicadas en niños desde los tres años, con valores de referencia disponibles desde los cinco ${ }^{1}$.

Otra de las pruebas utilizadas en la actualidad y que cuentan con una versión en español, es la prueba de escucha en ruido o HINT (hearing in noise test). Originalmente, esta prueba cuenta con 250 listas de oraciones que se encuentran divididas en 25 listados de 10 oraciones cada una, fonéticamente balanceadas $^{29}$. Las oraciones son presentadas a través de un altavoz ubicado a un metro del paciente y a $0^{\circ}$ azimuth (de frente). La evaluación considera cuatros condiciones de prueba: listado de palabras sin ruido competitivo, con ruido competitivo presentado al frente del paciente, con ruido a $90^{\circ}$ a la derecha y con ruido a $90^{\circ}$ a la izquierda. El ruido utilizado coincide espectralmente con las listas, y se presenta también a un metro de distancia del sujeto. En todas las condiciones, el ruido competitivo se presenta con un volumen constante de $65 \mathrm{~dB}$, disminuyendo o aumentando la intensidad de las oraciones en $2 \mathrm{~dB}$ hasta encontrar aquel SNR en el cual el paciente logra repetir de manera adecuada al menos el 50\% de las oraciones. Este método adaptativo reduce el efecto techo y/o suelo (ningún paciente puntuará $0 \%$ o $100 \%$ ) que es común encontrar en otras pruebas de este tipo y permite comparaciones de puntajes entre diferentes pacientes y diferentes condiciones para un mismo paciente. 
Si bien el HINT no corresponde a una prueba monoaural, ha ganado popularidad y aceptación a nivel internacional, habiéndose estandarizado en varios idiomas y convirtiéndose en una prueba comparable entre países. Actualmente, existen versiones disponibles en sueco $^{30}$, chino mandarín ${ }^{31}$, francés canadien$\mathrm{se}^{32}$, portugués brasileño ${ }^{33}$, entre otros. En español, se encuentran disponibles una versión castellana $^{34}$ y una versión latinoamericana ${ }^{35}$. Si bien en esta última versión el desarrollo de los materiales verbales se realizó en México, Colombia y Argentina, la adaptación contó con la participación de algunos centros nacionales, incluyendo la Clínica Las Condes y el Centro de Audición y Lenguaje Comunica.

A diferencia de otras categorías de pruebas, no se han encontrado reportes de sensibilidad/ especificidad, habiéndose utilizado principalmente para describir el rendimiento funcional en condiciones fisiológicas y patológicas. Sin embargo, se ha evidenciado que la prueba SSI-ICM es de gran utilidad para la detección de lesiones a nivel de tronco cerebral, principalmente en su porción más baja ${ }^{25,26}$. Se ha documentado, además, que la prueba PSI-ICM, utilizada en conjunto con una versión que utiliza ruido contralateral (PSI-CCM), permitiría diferenciar con gran precisión lesiones ocupantes de espacio en áreas auditivas corticales y en tronco cerebral (núcleo coclear, complejo olivar, leminisco lateral y colículo inferior) de otras áreas del sistema nervioso central que no involucran áreas auditivas ${ }^{36}$. Generalmente, se ha descrito un rendimiento deficiente en el oído contralateral a disfunciones o lesiones corticales, y patrones de rendimiento irregular en el caso de alteraciones de tronco cerebral ${ }^{1}$.

\section{Pruebas de habla de tiempo comprimido}

Las pruebas de habla de tiempo comprimido son aquellas que utilizan estímulos verbales comprimidos electrónicamente, descartando algunos de los segmentos de la señal, pero sin modificar la frecuencia fundamental $^{7}$. Estas pruebas fueron diseñadas para evaluar la capacidad del sistema auditivo de procesar rápidamente los cambios espectrales. Esta habilidad es necesaria, por ejemplo, al enfrentar a interlocutores que hablan más rápido de lo normal, o a interlocutores no nativos de la lengua del oyente, quienes modifican frecuentemente las velocidades normales de las palabras o fonemas. Esta compresión es realizada utilizando softwares de edición de audio para lograr una modificación solamente en la velocidad del estímulo verbal, sin alterar aspectos como frecuencia o intensidad. El grado de compresión se expresa como un porcentaje de la señal original que se elimina, por ejemplo, una compresión del $45 \%$ indica que el estímulo verbal estaría ocupando un 55\% del tiempo original y que se ha eliminado el $45 \%$ de la señal. Es importante destacar que el grado de compresión es la variable más influyente en la inteligibilidad del mensaje, pudiendo ser hasta un 60\% (al menos en inglés) para lograr una inteligibilidad satisfactoria en sujetos con audición normal ${ }^{37}$. Si bien se puede observar que al aumentar la compresión de manera gradual entre un $0 \%$ y un $60 \%$ se produce una variación paulatina en el rendimiento, no sería significativa, por lo que se sugiere para uso clínico una compresión de hasta un $60 \%{ }^{1}$.

A pesar de que la primera prueba de este tipo fue descrita en el año $1977^{38}$, tuvieron que pasar varias décadas hasta que fueron realmente incorporadas a la práctica clínica. Fue con el desarrollo de la prueba de oraciones comprimidas en tiempo o TCST (time compressed sentences test $)^{39}$ y su incorporación a las más recientes versiones del SCAN, tanto en su versión para niños, adolescente y adultos ${ }^{40,41}$, que este tipo de pruebas cobró relevancia clínica. El TCST consiste en tres subpruebas, cada una con sus respectivas listas de oraciones que el sujeto evaluado debe repetir correctamente, con tasas de compresión de $0 \%, 40 \%$ y $60 \%$ respectivamente. Otra prueba ampliamente utilizada es la que incluye el set de pruebas de evaluación del Centro Médico de Asuntos Veteranos ${ }^{42}$ en Estados Unidos. Esta grabación consta de cuatro listas de 50 monosílabos precedidos de frases, dos de ellas comprimidas a una tasa del $45 \%$ y otras dos a $65 \%$. Existe una versión alternativa de esta prueba incluida en la batería, que ocupa los mismos listados y tasas de compresión, pero a los listados se les agrega un grado de reverberancia de 0,3 segundos. Esto último para producir un efecto multiplicativo, es decir, una mayor dificultad 
en el reconocimiento del habla a través de una combinación de técnicas de degradación de la señal ${ }^{43}$. Sin embargo, esta última forma de degradación ha sido escasamente difundida en la clínica.

Para la población hispanohablante, existe una adaptación de la prueba original TCST ${ }^{44}$. Esta versión, con excepción del idioma, es idéntica a la original. Se compone de tres subpruebas: la primera está compuesta por 10 oraciones con una tasa de compresión de $0 \%$ utilizada como práctica, la segunda se compone de dos listados de 10 oraciones a una tasa de compresión del $40 \%$ y la tercera también utiliza dos listados de 10 oraciones, pero a una tasa de compresión del 60\%. La prueba se presenta a $55 \mathrm{~dB}$ HL si el sujeto evaluado tiene audición normal, o a una intensidad confortable si el sujeto tiene pérdida auditiva. La tarea del sujeto evaluado es repetir correctamente la oración, pudiendo asignarse hasta tres puntos por oración, un punto por cada parte repetida correctamente (sujeto, verbo y predicado). El resultado se consigna en porcentajes, por lo que cada segmento de las oraciones repetido correctamente equivale a un 3,3\%. En población pediátrica, el desempeño va mejorando sutilmente con la edad desde los siete años, donde el rendimiento promedio es de $93 \%$ a una tasa de compresión del $40 \%$ y de un $74 \%$ a una tasa de $60 \%$, hasta los once años donde el desempeño promedio es de un $96 \%$ a una tasa de $40 \%$ y de $86 \%$ a una tasa del $60 \%{ }^{44}$. En adultos, el rendimiento se tiende a estabilizar alrededor del $86 \%$ para tasas de hasta un $60 \%{ }^{7}$.

Se ha descrito que las pruebas de habla de tiempo comprimido tienen una sensibilidad de un $80 \%$ para lesiones o disfunciones de lóbulo temporal, 64\% para lesiones de tronco cerebral, $47 \%$ para lesiones vasculares de tronco y de un $67 \%$ para lesiones en el ángulo pontocerebeloso ${ }^{17}$. Además, en términos generales, se ha documentado que podrían ser particularmente útiles en aquellas lesiones que involucran de manera difusa la corteza auditiva primaria ${ }^{45}$. Al igual que en el resto de las pruebas, el rendimiento deficiente se presenta la mayoría de las veces en el oído contralateral a la lesión cortical, y dependiendo de las características, las lesiones de tronco pueden manifestarse en rendimientos deficientes ipsilaterales, contralaterales y bilaterales ${ }^{7}$.

\section{Consideraciones clínicas de las pruebas monoaurales de baja redundancia}

$\mathrm{Al}$ igual que para otros procesos auditivos centrales, las pérdidas auditivas periféricas podrían influenciar en distinta medida los mecanismos de MSC. En un estudio realizado en adultos mayores (controlando las variables como edad y cognición), se evidenció que una pérdida auditiva sensorioneural no afectaría el rendimiento en la prueba SIN, sin embargo, sí afectaría el rendimiento de pruebas FS, TCST y la SSI-ICM ${ }^{46}$. Esto llama especialmente la atención, ya que la prueba SIN y SSI-ICM, a pesar de pertenecer a la misma categoría de pruebas, no serían igualmente influenciadas por una pérdida auditiva periférica por lo que, podrían estar evaluando subprocesos sutilmente distintos, el grado de audibilidad afectaría de distintas maneras a cada prueba según las características acústicas que las diferencian (en este caso, el tipo de ruido competitivo) o el grado de redundancia extrínseca de cada prueba sería un factor que estaría interactuando con la hipoacusia al nivel de producir diferencias de rendimiento.

Las frecuencias afectadas por pérdidas auditivas también serían un factor influyente en el desempeño en estas pruebas, independiente del tipo y grado de pérdida. Se han comparado grupos de sujetos con pérdida en frecuencias agudas (sobre $3000 \mathrm{~Hz}$ ), pérdida en todas las frecuencias y sujetos con audición normal, evidenciando que aquellos con pérdida auditiva en todas las frecuencias presentan el rendimiento más bajo en las pruebas FS y TCST ${ }^{46}$. El rendimiento en la prueba FS es explicado debido a que utiliza filtros de pasa baja, por lo que es esperable que sujetos con hipoacusia en frecuencias graves tengan dificultades para completarlas. Por otro lado, la compresión de tiempo que ocurre en el TCST, estaría degradando algunos aspectos sutiles que enriquecen el habla normal, aspectos que se codificarían en mayor medida por las frecuencias graves.

En otro estudio ${ }^{47}$, se evidenció que un grupo de sujetos con pérdida auditiva en frecuencias agudas (de $3000 \mathrm{~Hz}$ en adelante) y con frecuencias graves normales, presentaban un rendimiento descendido en una prueba de FS. Si bien esto no es esperable, los autores hipotetizaron que las fibras nerviosas de fre- 
cuencias agudas tendrían un rol importante en la codificación de algunos aspectos que facilitarían la inteligibilidad del mensaje en este tipo de pruebas. Este hallazgo es además coherente con otro estudio de neuroimagen ${ }^{48}$, donde se ha concluido que el cerebro de sujetos con pérdidas auditivas en frecuencias agudas puede modificar algunas inervaciones de fibras nerviosas destinadas a frecuencias graves para compensar la alta frecuencia. Esta disminución en el procesamiento de frecuencias bajas, sumado a la composición espectral rica en componentes graves que tiene el ruido en general, contribuiría a las dificultades de habla en ruido de sujetos con hipoacusias de perfil descendente $y$, por ende, disminuiría el rendimiento en pruebas como el SSI-ICM.

En comparación con sujetos con audición normal, se ha evidenciado que pacientes con hipoacusia conductiva unilateral leve y moderada presentarían rendimientos significativamente inferiores en la prueba HINT, y este sería más bajo aún en aquellos sujetos con hipoacusia conductiva bilateral ${ }^{49}$. Un hallazgo similar se evidenció utilizando una prueba de habla en ruido de la Queensland University, donde un grupo de niños con hipoacusia conductiva bilateral presentó rendimientos más bajos que pares con audición normal ${ }^{50}$. Incluso, aquellos sujetos con hipoacusia conductiva unilateral y aquellos con hallazgos timpanométricos sugerentes de hipoacusia de conducción, pero con umbrales auditivos normales, también presentaron un rendimiento más bajo de lo esperado. Se ha podido evidenciar que pérdidas auditivas conductivas bilaterales transitorias, dejarían algún tipo de secuela en los procesos de MSC, demostrado en un rendimiento descendido en la prueba PSI-ICM en comparación con sujetos sin historial de problemas auditivos ${ }^{51}$. Se ha constatado que, independiente del tipo, las hipoacusias unilaterales en oído izquierdo presentarían más dificultades en la prueba SIN que aquellas en el oído derecho ${ }^{52}$, aunque esto no estaría del todo claro ${ }^{53}$.

A pesar de que en otro tipo de pruebas de procesamiento auditivo existiría un efecto de oído (mejor rendimiento de un oído por condiciones fisiológicas normales), en las pruebas monoaurales de habla de baja redundancia no se han reportado ventajas para ningún oído. En un estudio realizado por Abdo y cols. ${ }^{54}$, la prueba SIN no presentó diferencias significativas entre oídos en sujetos normales, sujetos con déficit atencional e hiperactividad y sujetos con dislexia. Algunas pruebas de habla de tiempo comprimido y particularmente la prueba TCST ${ }^{55,56}$, tampoco han evidenciado tener algún rendimiento superior en algún oído en sujetos normales, e incluso para sujetos con alteraciones del procesamiento auditivo. La prueba FS tampoco ha mostrado diferencias entre oídos, sin embargo, sí se identificó una diferencia entre el primer oído en ser evaluado y el segundo, presentando este último un mejor desempeño sin importar si se trataba del oído izquierdo o derecho ${ }^{15}$. Este hallazgo también ha sido evidenciado en la prueba $\operatorname{SIN}^{15}$ y en la prueba TCST $^{55}$, atribuyéndose a un efecto de aprendizaje y, por ende, a un aumento de la redundancia extrínseca ${ }^{57}$. Solamente se encontró un estudio en el cual se evidenció una ventaja significativa del oído derecho en condición de primer oído evaluado, sin embargo, los autores atribuyeron esto a temas atencionales debido a que las asimetrías funcionales no se podrían evidenciar en pruebas monoaurales ${ }^{58}$.

Al interpretar este tipo de pruebas, el género no sería un factor influyente. Se ha demostrado que no existen diferencias en el rendimiento de hombres y mujeres tanto para las pruebas SIN y FS ${ }^{15}$, como para la prueba TCST $^{59}$. Sin embargo, sí se debiese considerar la influencia que pueden tener factores como el nivel y tipo de escolaridad, nivel socioeconómico y la vulnerabilidad social de los sujetos evaluados. Los hallazgos del estudio de Borges y cols.$^{60}$ indican que niños y adolescentes en situación de calle o abandono, abusados física o sexualmente y con periodos prolongados de deserción escolar, mostraron rendimientos significativamente menores en una prueba no identificada de habla en ruido. También se ha podido comprobar que niños de colegios públicos tendrían un rendimiento menor al de niños de colegios privados en la prueba PSI-ICM, atribuyendo estos resultados a diferencias socioculturales ${ }^{51}$. La exposición a experiencias auditivas culturalmente enriquecido como la formación o entrenamiento musical para niños pequeños, ha demostrado mejorar el rendimiento en algunas pruebas. Así se demostró en un estudio que comparó el rendimiento de la prueba HINT en dos 
grupos de niños de educación primaria con bajo nivel socioeconómico, uno de los cuales estuvo expuesto durante mayor tiempo a actividades de formación musical y que justamente obtuvo mejores resultados en la prueba ${ }^{61}$. En sujetos universitarios se comprobó, además, que el tiempo de exposición a actividades de formación musical se correlaciona de manera significativa con el rendimiento de pruebas de habla en ruido como el HINT y el SIN ${ }^{62}$.

Al momento de la revisión, no se han encontrado estudios publicados en los que se haya utilizado alguna prueba monoaural de habla de baja redundancia de aplicación clínica asistida de audífonos o implante coclear. La gran mayoría de los estudios que documentan los procesos auditivos de MSC en esta población han utilizado sus propias pruebas de laboratorio y se han enfocado casi exclusivamente en la percepción del habla en ruido por ser una de las mayores dificultades de usuarios de ayudas auditivas ${ }^{63}$. Sin embargo, vale la pena mencionar que los usuarios de implante coclear han demostrado un rendimiento significativamente menor en tareas de habla en ruido en comparación con pares de audición normal, tanto niños ${ }^{64}$ como adultos $^{65}$. Además, en el mismo tipo de tareas, no se han encontrado diferencias significativas entre usuarios de implante coclear y audífonos ${ }^{64}$. En relación con estos últimos, algunos estudios sugieren que las pruebas de habla en ruido podrían ser de utilidad antes de aparatar audífonos, permitiendo elegir mejores configuraciones, predecir la adherencia y el rendimiento en situaciones reales, además de poder manejar de manera más eficiente las expectativas del paciente ${ }^{66}$.

En sujetos con implante coclear bilateral, se han descrito mejores rendimientos de habla en ruido con ambos implantes encendidos que solo con uno de ellos ${ }^{67}$. Estos resultados podrían ser explicados por tres efectos de la audición binaural en la percepción del habla en ruido: el efecto sombra de la cabeza, el efecto de sumación binaural y el efecto de silenciador binaura ${ }^{68}$. El primero de ellos ocurre cuando la cabeza produce un efecto de atenuación del ruido para el oído más alejado de su fuente, produciendo un mejor SNR. El segundo hace referencia a un aumento en la sonoridad (hasta $3 \mathrm{~dB}$ HL más que en audición monoaural), una mayor sensibilidad a las diferencias en intensidad y frecuencia cuando ambos oídos perciben la misma señal. La tercera se refiere a una disminución del ruido de fondo que realiza el SNAC a través del procesamiento de diferencias de tiempo, amplitud y características espectrales que provienen de ambos oídos. Aunque estos efectos son importantes para la percepción de habla en ruido, estos no corresponden a procesos de MSC, sino que serían más bien procesos de separación binaural e integración binaural ${ }^{2,3}$, por lo que escapan al alcance de esta revisión.

\section{Conclusión}

La separación y el cierre monoaural son habilidades del procesamiento auditivo que interactúan con factores acústicos, lingüísticos, cognitivos y contextuales. Sus mecanismos permiten mantener interacciones comunicativas significativas en ambientes reales, con condiciones acústicas adversas, ruido competitivo e interlocutores no ideales. En la actualidad, existen tres tipos de pruebas para su evaluación clínica que utilizan distintas formas de degradación de la redundancia extrínseca: pruebas de habla filtrada de pasa baja, pruebas de habla en ruido y pruebas de habla de tiempo comprimido. Las más utilizadas en el último tiempo han sido las pruebas de habla en ruido, ya que permitirían simular de mejor manera ambientes reales, sin embargo, los tres tipos de pruebas han documentado su utilidad. Independiente del tipo de prueba utilizada, se sugiere que todas las pruebas de percepción de habla, incluida las pruebas clásicas como la logoaudiometría, deberían interpretarse en función de la redundancia del paradigma de evaluación. La reducción de la redundancia extrínseca permite realizar una mejor aproximación al sistema auditivo propiamente tal y mejora su sensibilidad para detectar dificultades auditivas. Sin embargo, en la medida que aumenta la sensibilidad, se reduce proporcionalmente la validez ecológica de la prueba y, con ello, la posibilidad de aproximarnos al rendimiento del sujeto en contextos cotidianos y reales.

Se debe tener en consideración que algunas de las pruebas de una misma categoría se ven influenciadas de distinta manera por pérdidas 
auditivas periféricas, por lo que surge la duda sobre si efectivamente estos procesos centrales se ven afectados por tiempos de deprivación auditiva, o las características acústicas de estas pruebas provocarían per se un rendimiento deficiente en sujetos con hipoacusia. No existen estudios en los cuales se comparen distintas pruebas de una categoría, sin embargo, se debe cautelar su interpretación debido a que incluso sus diferencias más sutiles (por ejemplo, el tipo de ruido, el material verbal o los SNR en el caso de las pruebas de habla en ruido) podrían ocasionar diferencias de rendimiento, por evaluar subprocesos diferentes o por variar los niveles de redundancia requeridos para completar la tarea solicitada. Además, el rendimiento en estas pruebas podría estar influenciado por factores no audiológicos, como la escolaridad, nivel socioeconómico y otros determinantes sociales de la salud. Se sugiere la obtención de valores referenciales considerando estos y otros aspectos reportados en la literatura que podrían influenciar los resultados de estas pruebas.

\section{Bibliografía}

1. Bellis T. Assessment and Management of Central Auditory Processing Disorders in the Educational Setting: From Science to Practice (Second). 2011; San Diego, CA: Plural Publishing.

2. Schow R, Seikel A, Chermak G, Berent M. Measures: ASHA 1996 Revisited. Am J Audiol. 2000;9(2):63-68.

3. Domitz DM, Schow RL. A new CAPD battery-multiple auditory processing assessment: factor analysis and comparisons with SCAN. Am J Audiol. 2000;9(2):101-111. doi: 10.1044/10590889(2000/012).

4. American Speech-Language-Hearing Association. Central auditory processing: current status of research and implications for clinical practice [Technical Report]1996. Recuperado de www.asha.org/policy

5. American Speech-Language-Hearing Association. (Central) Auditory Processing Disorders [Technical Report] 2005. Recuperado de www.asha.org/policy

6. Geffner D, Ross-Swain D. Auditory Processing Disorders Assessment, Management, and Treatment (Second Ed.).2013. San Diego, CA: Plural Publishing.

7. Krishnamurti S. Monaural low-redundancy speech tests. En F Musiek \& G. Chermak (Eds.), Handbook of Central Auditory Processing Disorder: Volume I Auditory Neuroscience and Diagnosis 2014; (Second Ed., pp. 349-368). San Diego, CA: Plural Publishing.
8. Tirapu J. La evaluación neuropsicológica. Psychosocial Intervention. 2007;16(2):189-211.

9. Lawson G, Peterson M. Speech Audiometry. 2011. San Diego, CA: Plural Publishing.

10. Bocca E, Calearo C, Cassinari V. A new method for testing hearing in temporal lobe tumours; preliminary report. Acta Otolaryngol. 1954;44(3):219221. doi: $10.3109 / 00016485409128700$.

11. Bocca E, Calearo C, Cassinari V, Migliavacca F. Testing "cortical" hearing in temporal lobe tumours. Acta Otolaryngol. 1955;45(4):289-304. doi: 10.3109/00016485509124282.

12. Willeford, J. Assessing central auditory behavior in children: A test battery approach. En R. Keith (Ed.), Central auditory dysfunction 1977. (pp. 43-72). New York, NY: Grune \& Stratton.

13. Keith RW. Development and standardization of SCAN-C Test for Auditory Processing Disorders in Children. J Am Acad Audiol. 2000;11(8):438-445.

14. Keith R. SCAN 3 for Adolescents \& Adults Technical Report. 2012. Pearson Education.

15. Fuente A, McPherson B. Auditory processing tests for Spanish-speaking adults: an initial study. Int J Audiol. 2006;45(11):645-659. doi: 10.1080/14992020600937238

16. Rosenblüt B, de la Cruz J. Listas de palabras en español para pruebas de discriminación. Rev Otorrinolaringol Cir Cabeza Cuello. 1962; 22(2):37-49.

17. Karlsson AK, Rosenhall U. Clinical application of distorted speech audiometry. Scand Audiol. 1995;24(3):155-160. doi: 10.3109/01050399509047529.

18. Musiek FE, Chermak GD, Weihing J, Zappulla M, Nagle S. Diagnostic accuracy of established central auditory processing test batteries in patients with documented brain lesions. J Am Acad Audiol. 2011;22(6):342-358. doi: 10.3766/jaaa.22.6.4.

19. American Academy of Audiology. Clinical Practice Guidelines: Diagnosis, Treatment and Management of Children and Adults with Central Auditory Processing Disorder. 2010. Recuperado de https:// www.audiology.org/publications-resources/ document-library/central-auditory-processingdisorder.

20. Miller GA. The masking of speech. Psychol Bull. 1947;44(2):105-129. doi: 10.1037/h0055960.

21. Morales-Garcia C, Poole JP. Masked speech audiometry in central deafness. Acta Otolaryngol. 1972;74(5):307-316. doi: 10.3109/00016487209128456.

22. Heilman KM, Hammer LC, Wilder BJ. An audiometric defect in temporal lobe dysfunction. Neurology. 1973;23(4):384-386. doi: 10.1212/wnl.23.4.384.

23. Wilson RH. Development of a speech-in-multitalkerbabble paradigm to assess word-recognition performance [published correction appears in J Am 
Acad Audiol. 2010 Jan;21(1):66]. J Am Acad Audiol. 2003;14(9):453-470.

24. Keith RW. Development and standardization of SCAN-A: test of auditory processing disorders in adolescents and adults. J Am Acad Audiol. 1995;6(4):286-292.

25. Jerger J, Jerger S. Auditory findings in brain stem disorders. Arch Otolaryngol. 1974;99(5):342-350. doi: 10.1001/archotol.1974.00780030354006

26. Jerger J, Jerger S. Clinical validity of central auditory tests. Scand Audiol. 1975;4(3):147-163.

27. Jerger S, Jerger J. Pediatric Speech Intelligibility Test: Manual for administration. 1984. St. Louis, MO: Auditec.

28. Benítez L, Speaks C. Development of a Synthetic Sentence Identification Task in Spanish. J Acoust Soc Am. 1968;44(1):398.

29. Nilsson M, Soli SD, Sullivan JA. Development of the Hearing in Noise Test for the measurement of speech reception thresholds in quiet and in noise. J Acoust Soc Am. 1994;95(2):1085-1099. doi: $10.1121 / 1.408469$.

30. Hällgren M, Larsby B, Arlinger S. A Swedish version of the Hearing In Noise Test (HINT) for measurement of speech recognition. Int J Audiol. 2006;45(4):227-237. doi: 10.1080/14992020500429583.

31. Wong LL, Soli SD, Liu S, Han N, Huang MW. Development of the Mandarin Hearing in Noise Test (MHINT). Ear Hear. 2007;28(2 Suppl):70S-74S. doi: 10.1097/AUD.0b013e31803154d0.

32. Vaillancourt V, Laroche C, Mayer C, et al. The Canadian French hearing in noise test. Int J Audiol. 2008;47(6):383-385. doi: 10.1080/14992020802055300.

33. Bevilacqua MC, Banhara MR, Da Costa EA, Vignoly $\mathrm{AB}$, Alvarenga KF. The Brazilian Portuguese hearing in noise test. Int J Audiol. 2008;47(6):364-365. doi: 10.1080/14992020701870205.

34. Huarte A. The Castilian Spanish hearing in noise test. Int J Audiol. 2008;47(6):369-370. doi: 10.1080/14992020801908269.

35. Baron de Otero C, Brik G, Flores L, Ortiz S, Abdala C. The Latin American Spanish hearing in noise test. Int J Audiol. 2008;47(6):362-363. doi: 10.1080/14992020802060888.

36. Jerger $\mathrm{S}$. Validation of the pediatric speech intelligibility test in children with central nervous system lesions. Audiology. 1987;26(5):298-311. doi: 10.3109/00206098709081557.

37. Wilson RH, Preece JP, Salamon DL, Sperry JL, Bornstein SP. Effects of time compression and time compression plus reverberation on the intelligibility of Northwestern University Auditory Test No. 6. J Am Acad Audiol. 1994;5(4):269-277.

38. Beasley D, Freeman B. Timealtered speech as a measure of central auditory processing. 1977. En R.
Keith (Ed.), Central auditory dysfunction (pp. 129176). New York, NY: Grune \& Stratton.

39. Keith R. Standarization of the Time Compressed Sentence Test. Journal of Educational Audiology. 2002; 10:15-20.

40. Keith R. SCAN-3:A. Tests for Auditory Processing Disorders in Adolescents and Adults. 2009a. Upper Saddle River, NJ: Pearson.

41. Keith R. SCAN-3:C. Tests for Auditory Processing Disorders in Children. 2009b. Upper Saddle River, NJ: Pearson.

42. Department of Veterans Affairs. Tonal and speech materials for auditory perceptual assessment, Disc 2.0. 2011. Mountain Home, TN: VA Medical Center.

43. HARRIS JD. Combinations of distortion in speech. The twenty-five per cent safety factor by multiple-cueing. Arch Otolaryngol. 1960;72:227-232. doi: 10.1001/archotol.1960.00740010232014.

44. Keith R. Spanish Time Compressed Sentence Test. 2006. St. Louis, MO: Auditec.

45. Mueller G, Beck W, Sedge R. Comparision of the efficiency of cortical level speech test. Semin Hear. 1987; 8(3):279-298.

46. Cox LC, McCoy SL, Tun PA, Wingfield A. Monotic auditory processing disorder tests in the older adult population. J Am Acad Audiol. 2008;19(4):293-308. doi: 10.3766/jaaa.19.4.3.

47. Horwitz AR, Dubno JR, Ahlstrom JB. Recognition of low-pass-filtered consonants in noise with normal and impaired high-frequency hearing. J Acoust Soc Am. 2002;111(1 Pt 1):409-416. doi: 10.1121/1.1427357.

48. Lockwood AH, Salvi RJ, Coad ML, et al. The functional anatomy of the normal human auditory system: responses to 0.5 and $4.0 \mathrm{kHz}$ tones at varied intensities. Cereb Cortex. 1999;9(1):65-76. doi: 10.1093/cercor/9.1.65.

49. Hsieh DL, Lin KN, Ho JH, Liu TC. Hearing in noise test in subjects with conductive hearing loss. J Formos Med Assoc. 2009;108(12):937-942. doi: 10.1016/ S0929-6646(10)60006-X.

50. Keogh T, Kei J, Driscoll C, Khan A. Children with minimal conductive hearing impairment: speech comprehension in noise. Audiol Neurootol. 2010;15(1):27-35. doi: 10.1159/000218360.

51. Borges LR, Paschoal JR, Colella-Santos MF. (Central) auditory processing: the impact of otitis media. Clinics (Sao Paulo). 2013;68(7):954-959. doi: 10.6061/clinics/2013(07)11.

52. Nishihata R, Vieira R, Pereira L, Chiari M. Temporal processing, localization and auditory closure in individuals with unilateral hearing loss. Rev Soc Bras Fonoaudiol. 2012;17(3):266-273.

53. Hartvig Jensen J, Johansen PA, Børre S. Unilateral sensorineural hearing loss in children and auditory performance with respect to right/left ear differences. Br J Audiol. 1989;23(3):207-213. doi: 10.3109/03005368909076501. 


\section{ARTÍCULO DE REVISIÓN}

54. Abdo A, Murphy C, Schochat E. Habilidades auditivas em crianças com dislexia e transtorno do déficit de atenção e hiperatividade. Pro-Fono. 2010; 22(1):25-30.

55. Rabelo CM, Schochat E. Time-compressed speech test in Brazilian Portuguese. Clinics (Sao Paulo). 2007;62(3):261-272. doi: 10.1590/s180759322007000300010.

56. Arceno RS, Scharlach RC. Time-compressed speech test in the elderly. Teste de fala comprimida em idosos. Codas. 2017;29(5):e20160243.

Published 2017 Sep 28. doi: 10.1590/2317$1782 / 20172016243$.

57. Marcotti A, Aguilar G, Farías C, Galaz J, Iturriaga F. Obtención de valores referenciales para la prueba de habla filtrada en una población de adultos chilenos. En Libro del LXXV Congreso Chileno de Otorrinolaringología, 2018, (p. 73). Santa Cruz: Sociedad Chilena de Otorrinolaringología.

58. Rabelo CM, Rocha-Muniz CN, Schochat E. Ongoing maturation in the time-compressed speech test. Clinics (Sao Paulo). 2018;73:e407. Published 2018 Nov 29. doi: 10.6061/clinics/2018/e407.

59. Padilha FYOMM, Pinheiro MMC. Study on the application of the time-compressed speech in children. Estudo do teste de fala comprimida em crianças. Codas. 2017;29(5):e20160239. doi: 10.1590/2317-1782/20172016239.

60. Murphy CF, Pontes F, Stivanin L, Picoli E, Schochat E. Auditory processing in children and adolescents in situations of risk and vulnerability. Sao Paulo Med J. 2012;130(3):151-8. doi: 10.1590/s151631802012000300004 .
61. Slater J, Skoe E, Strait DL, O'Connell S, Thompson E, Kraus N. Music training improves speech-innoise perception: Longitudinal evidence from a community-based music program. Behav Brain Res. 2015;291:244-252. doi: 10.1016/j.bbr.2015.05.026.

62. Ruggles DR, Freyman RL, Oxenham AJ. Influence of musical training on understanding voiced and whispered speech in noise. PLoS One. 2014;9(1):e86980. doi: 10.1371/journal.pone.0086980.

63. Ciorba A, Zattara S, Loroni G, Prosser S. Quantitative enhancement of speech in noise through a wireless equipped hearing aid. Acta Otorhinolaryngol Ital. 2014;34(1):50-53.

64. Caldwell A, Nittrouer S. Speech perception in noise by children with cochlear implants. J Speech Lang Hear Res. 2013;56(1):13-30. doi: 10.1044/10924388(2012/11-0338).

65. Nascimento LT, Bevilacqua MC. Evaluation of speech perception in noise in cochlear implanted adults. Braz J Otorhinolaryngol. 2005;71(4):432-438. doi: 10.1016/ s1808-8694(15)31195-2.

66. Taylor B. The Acceptable Noise Level Test as a predictor of real-world hearing aid benefit. Hearing Journal. 2008;61(9):39-42.

67. Almeida GFL, Martins MF, Costa LBAD, Costa OAD, Martinho de Carvalho AC. Sequential bilateral cochlear implant: results in children and adolescents. Braz J Otorhinolaryngol. 2019;85(6):774779. doi: 10.1016/j.bjorl.2018.07.008.

68. Brown KD, Balkany TJ. Benefits of bilateral cochlear implantation: a review. Curr Opin Otolaryngol Head Neck Surg. 2007;15(5):315-318. doi: 10.1097/ MOO.0b013e3282ef3d3e. 